\title{
COMPRESSIVE SENSING IMAGE FUSION BASED ON PARTICLE SWARM OPTIMIZATION ALGORITHM
}

\author{
Xianhu $\mathrm{Li}^{\mathrm{a}}$, Jingguo $\mathrm{Lv}^{\mathrm{b}}{ }^{*}$, Shan Jiang ${ }^{\mathrm{c}}$, Huilin Zhou ${ }^{\mathrm{d}}$ \\ a, school of geomatics and urban spatial information, Beijing University of Civil Engineering and Architecture, \\ 102616 Beijing, China - 1003240752@qq.com \\ ${ }^{\mathrm{b}}$ school of geomatics and urban spatial information, Beijing University of Civil Engineering and Architecture, \\ 102616 Beijing, China - lvjingguo@bucea.edu.cn \\ ${ }^{\mathrm{c}}$ school of geomatics and urban spatial information, Beijing University of Civil Engineering and Architecture, \\ 102616 Beijing, China - 379768614@qq.com \\ d school of medicine, Shanghai Jiao Tong University, 200240 Shanghai, China - 2667679904@qq.com
}

KEY WORDS: Particle swarm optimization, Compressive sensing, HIS-CS image fusion, Fusion coefficient, self-adaptability

\begin{abstract}
:
In order to solve the problem that the spatial matching is difficult and the spectral distortion is large in traditional pixel-level image fusion algorithm. We propose a new method of image fusion that utilizes HIS transformation and the recently developed theory of compressive sensing that is called HIS-CS image fusion. In this algorithm, the particle swarm optimization algorithm is used to select the fusion coefficient $\omega$. In the iterative process, the image fusion coefficient $\omega$ is taken as particle, and the optimal value is obtained by combining the optimal objective function. Then we use the compression-aware weighted fusion algorithm for remote sensing image fusion, taking the coefficient $\omega$ as the weight value. The algorithm ensures the optimal selection of fusion effect with a certain degree of self-adaptability. To evaluate the fused images, this paper uses five kinds of index parameters such as Entropy, Standard Deviation, Average Gradient, Degree of Distortion and Peak Signal-to-Noise Ratio. The experimental results show that the image fusion effect of the algorithm in this paper is better than that of traditional methods.
\end{abstract}

\section{PREFACE}

Image fusion is an optimization method for spatial information and spectral information, which eliminates suppression and redundant information, highlights the information needed, and provides a more targeted and accurate images in various research fields (Wang X, 2015). Currently, the fusion methods include weighted average fusion, HSI transform algorithm, Brovey transform, principal component analysis algorithm, Gram-Schmidt Spectral Sharpening, Pan-Sharpening, wavelet fusion algorithm etc.. Among these methods, HIS is one of the most widely used image fusion algorithms (Yang $\mathrm{J}$, 2014). The traditional HIS image fusion algorithm can improve the spatial resolution of the fusion image to a certain extent, but it also causes spectral distortion because of the loss of the spectral information (Jianwei Liu, 2013). So the traditional HIS transform algorithm can't get the ideal fusion effect in spectral information and spatial resolution at the same time (TU Te-ming,2001, Zhang Y, 2012). In recent years, some scholars have proposed the fusion methods based on compressive sensing (CS) (Guo J, 2013). Alin Achim (2008) proposed a new image fusion algorithm in the compressive domain by using an improved sampling pattern; the study demonstrates that CS-based image fusion has a number of perceived advantages in comparison with image fusion in the

\footnotetext{
* Address all correspondence to: Jingguo Lv, E-mail:
} lvjingguo@bucea.edu.cn multi-resolution (MR) domain (Wan T, 2008). Then Atul Diveker et al. improves the compression-aware image fusion algorithm by creating a dictionary that relates high resolution image patches from a panchromatic image to the corresponding filtered low resolution versions. Any pair of co-occurring high and low resolution patches with similar statistical properties to the patches in the dictionary is sparse with respect to the principal component bases. The result shows that it can reduce the spectral distortion of the fused image and produces results superior to standard fusion methods such as the Brovey transform and principal component analysis (Atul. Divekar, 2009). Yang (2015) proposed an image fusion method based on block-based compressed sensing (BCS) that can reduce the storage requirements and reconstruction cost to a certain extent (Senlin Yang, 2015). Considering the advantages and disadvantages of HIS transform and compressive sensing (CS), we propose the HIS-CS image fusion algorithm. The key problem in HIS-CS algorithm is how to choose the appropriate fusion coefficient $\omega$. In this paper, we use the particle swarm optimization algorithm to select the appropriate coefficient $\omega$.

In the iterative process of particle swarm optimization algorithm, the image fusion coefficient $\omega$ is used as the particle, and the information entropy and the average gradient function are used as the optimization objective function. The goal is to make the fusion measurement value maximize the information entropy and the average gradient value so as to achieve the optimal value of the fusion coefficient under the 
function. In the iterative process of particle swarm optimization algorithm, the image fusion coefficient $\omega$ is used as the particle, and the functions of entropy and the average gradient are taken as the optimization objective function. The goal is to get one $\omega$, which can maximize the value of functions of entropy and the average gradient. Then we take this optimal value as a weight coefficient during the use of weighted fusion rules for image fusion. This method can not only ensure the optimization of the fusion effect, but also make the fusion algorithm have a certain degree of adaptability.

\section{Compressive Sensing(CS)}

Candes and Donoho put forward CS theory in 2006 (Donoho D, 2006). And then it had a rapid development on the bases of the existing theories such as signal reconciliation analysis, time-frequency analysis, statistical probability theory, matrix analysis, functional analysis, statistical probability theory and so on (Gesen Zhang, 2012). Compressive Sensing can achieve compression at the same time as sampling. The flow chart of compressive sensing is as shown in Figure 1.

Step 2 Step 3

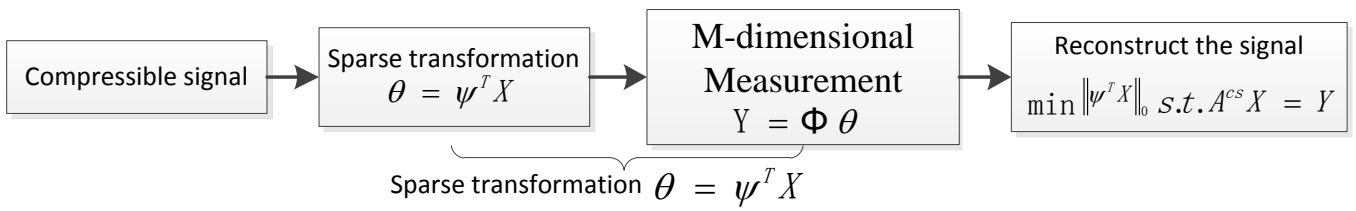

Figure 1 The flow chart of Compressive Sensing

Compressive sensing explores the essential structure of the signal from the overall situation, which can get rid of the links between signal frequency and other physical measurement (Zhang Y, 2012). As long as the signal has a compressible sparse domain, it can be linearly projected onto a low-dimension observation vectors using a measurement matrix that is non-coherent with the transformation matrix. The sample value in projection space contains enough information. So we can use the sparse optimization theory to accurately reconstruct the original signal from a small number of sample values with high probability.

The theory of compression sensing mainly includes the sparse representation of the signal, the measurement matrix design and reconstruction algorithm selection. One of the advantages is that it gathers the steps of data collection and data sample into one. The data is compressed at the same time as the signal acquisition, and the cost in the signal acquisition and processing is greatly reduced.

The process of compressive sensing is as follows:

Step 1: If the signal $X \in R^{N}$ is sparsely on an orthogonal basis $\psi$, then we can find the transform coefficients by $\theta=\psi^{T} X$. $\theta$ is the equivalent or approximation representation of signal $X$ in the $\psi$ space domain.

Step 2: We design a $M \times N$ measurement matrix

$\phi$, which has no relevance with the orthogonal basis

$\psi$. Then we can obtain the measurements

$Y=\phi \theta=\phi \psi^{T} X$

process can also be expressed as the adaptive

observation of signal $X$ under the matrix

$Y=A^{c s} X \quad\left(A^{c S}=\phi \psi^{T}\right), A^{c s}$ is called the information operator of Compressive Sensing.

Step 3: The sparse signal $x$ can be recovered using measurements to solve (1) if the projection matrix satisfies the restricted isometry property (RIP).

$$
\begin{gathered}
\min \left\|\psi^{T} X\right\|_{o} \text { s.t. } \\
Y=A^{c S} X \approx \phi \psi^{T} X
\end{gathered}
$$

The superiority of CS is that the sampling quantity is far less than the amount of data obtained by the traditional Nyquist sampling method, breaking through the limitation of the Nyquist sampling theorem.

\section{Particle Swarm Optimization Algorithm}

Particle swarm optimization (PSO) is an evolutionary computation. The basic thought is to find the optimal solution through the cooperation and information sharing among the individuals in the swarm (Yujie Xu, 2013). In this algorithm, the bird is abstracted as a point (particle) without volume and mass, and is extended to the $\mathrm{N}$-dimensional space. The position vector of the particle $\mathrm{i}$ in the $\mathrm{N}$-dimensional space is expressed as $X_{i}=\left(x_{1}, X_{2}, \mathrm{~L}, X_{N}\right)$, and the vector of the flight velocity is expressed as $V_{i}=\left(V_{1}, V_{2}, \mathrm{~L}, V_{N}\right)$. Each particle has a fitness value determined by the objective function, and also knows it's current position $X_{i}$ and the best position has been found. This process can be seen as the particle's own flight experience. In addition, each particle knows the best position gbest (which is the best value of all pbest), which is currently found by the whole group. This process can be seen as the flight experience of the swarm. Particles in the swarm determine the direction and velocity of the next step by the best experience of their peers in the group. In this process, the Particle Swarm Optimization (PSO) algorithm is first initialized into a group of random particles (random solutions), and then obtained the optimal solution by iteration. In each iteration process, the particles update their position and velocity through two extreme (pbest, gbest).

$$
\begin{aligned}
& V_{i}=V_{i}+c_{1} \times \operatorname{rand}() \times\left(\text { pbest }_{i}-X_{i}\right) \\
& +c_{2} \times \operatorname{rand}() \times\left(\text { gbest }_{i}-X_{i}\right)
\end{aligned}
$$




$$
X_{i}=X_{i}+V_{i}
$$

In formulas (2) and (3), $i=1,2,3 \mathrm{~L}, N$; and $N$ is the total number of particles in the swarm; $V_{i}$ is the velocity of the particle; $\operatorname{rand}()$ is the random number between 0 and $1 ; X_{i}$ is the current position of the particle; $c_{1}$ and $c_{2}$ are learning factors, usually $c_{1}=c_{2}=2$;

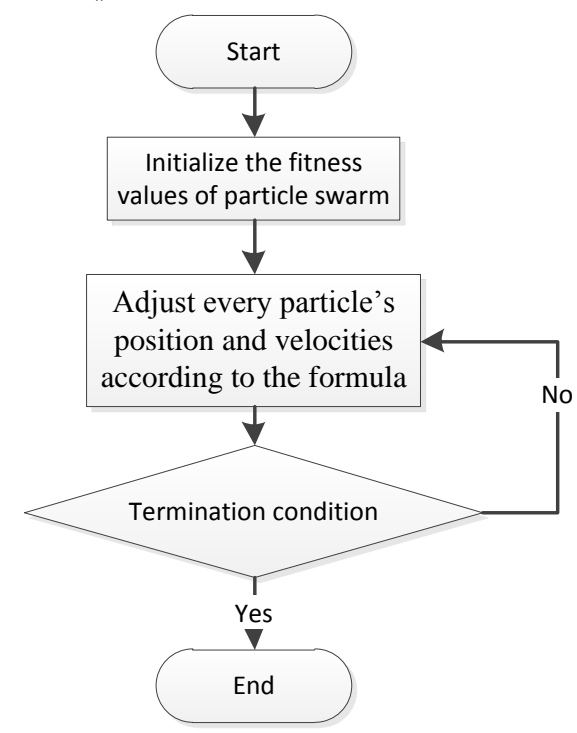

Figure 2 The flow chart of Particle Swarm Optimization algorithm

The process of Particle swarm optimization (PSO) is as follows:

Step 1: Initialize a group of particles (the size is N), including random positions and velocities;

Step 2: Evaluate the fitness of each particle;

Step 3: The current fitness value of each particle is compared with its best position (pbest) ever found, if the current fitness value is better, then its current fitness value will be the best position (pbest);

Step 4: The current fitness value of each particle is compared with the swarm's best position (gbest) ever found, if the current fitness value is better, then its current fitness value will be the swarm's best position (gbest);

Step 5: Adjust every particle's position and velocities according to the formula (2), (3)

Step 6: Check it out whether the results meet the end condition, if so then finish, or turn to step 2;

The iteration termination condition is selected according to the specific problem. Generally we select the maximum number of iterations $G_{k}$, or the optimal position to meet the minimum adaptation threshold currently found by the particle swarm(Jianyong Li, 2004).

\section{HIS - CS Fusion Algorithm Based on Particle Swarm Optimization Algorithm}

The advantage of HIS image fusion is that it can improve the spatial resolution obviously, and Compressive sensing (CS) can remain the spectral information better. In this paper, HIS-CS image fusion algorithm based on particle swarm optimization (PSO) is proposed utilizes the advantages of the two algorithm. The particle swarm optimization algorithm is used to determine the image fusion weight coefficient $\omega$ in the proposed method, which can avoids the problem that the experience value used in traditional methods can't be adjusted adaptively with different characteristics of different images. In this method, the particle swarm optimization algorithm is used to select the fusion parameter $\omega$. In the iterative process, the image fusion coefficient $\omega$ is used as particle; the functions of information entropy and the average gradient are taken as the optimization objective function, which goal is to get the maximum values of the information entropy and the average gradient. So we can get the optimal value under the defined optimization objective function. The optimal value is taken as the target fusion weight coefficient used in the weighted fusion rule in HIS-CS image fusion. The method can guarantee the optimization of the fusion effect and make the fusion method have a certain degree of adaptability.

The process of HIS-CS image fusion based on the particle swarm optimization algorithm is as shown if Figure 3: 


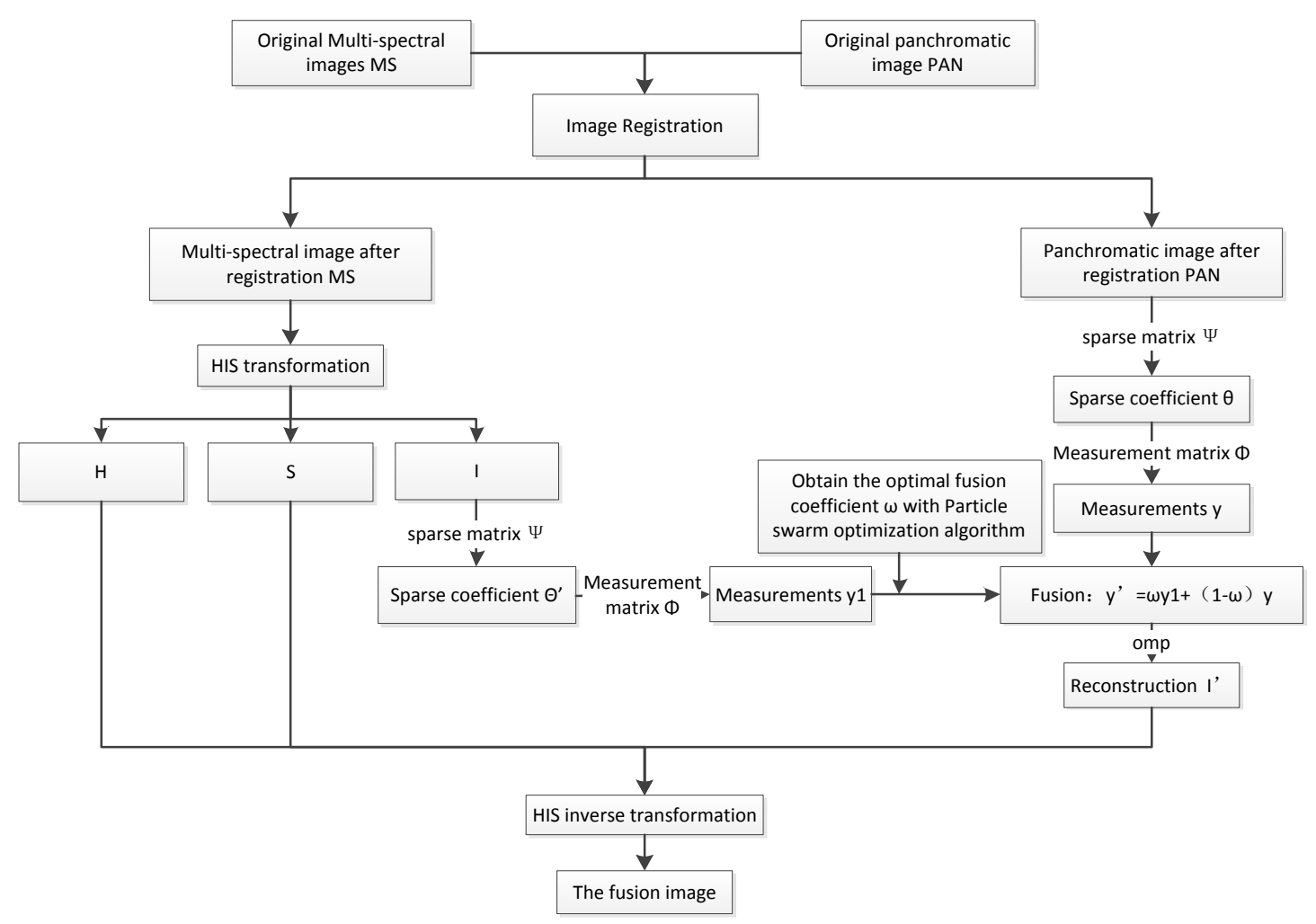

Figure 3 The flow chart of HIS - CS image fusion algorithm based on particle swarm optimization algorithm

The process of HIS-CS image fusion based on Particle swarm optimization (PSO) is as follows:

Step 1: Raw image processing: The high resolution image is meshed with the multi-spectral image, we can obtain two images with high spatial information matching. Then reshape the image according to the specific research area.

Step 2: The multi-spectral image with registration is transformed by HIS transformation, then we can get Three relatively independent components H (Hue), I (Intensity), S (Saturation).

Step 3: Then we get the measurement matrix $y 1$ and $y$ of panchromatic image PAN and I component got in step 2 by compressive sensing algorithm.

Step 4: The image measurements are fused:

$$
y^{\prime}=\omega y 1+(1-\omega) y, \omega \in(0,1) \text {; }
$$

Step 5: The particle swarm optimization algorithm is used to determine the image fusion weight coefficient $\omega$. In the iterative process, the image fusion

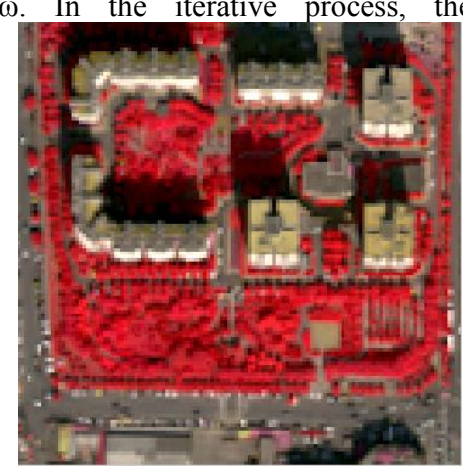

(a) Original Image

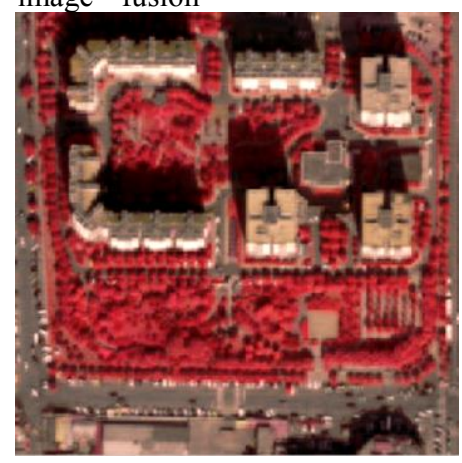

(b) PCA fusion coefficient $\omega$ is used as particle; the functions of information entropy and the average gradient are taken as the optimization objective function, which goal is to get the maximum values of the information entropy and the average gradient

Step 6: Reconstruct the image from the fused compressive measurements by OMP (orthogonal matching tracking). Then matched it with I component got in step 2 as a new intensity component $I^{\prime}$;

Step 7: Combining the new intensity component with $\mathrm{H}, \mathrm{S}$ components, we can use HIS inverse transformation to get the fusion image finally.

In this paper, the algorithm is validated by using the combination of WorldView panchromatic image and multi-spectral image, and then compared with the fusion effect of traditional methods, such as HIS transformation, PCA fusion, Compressive sensing image fusion, non-adaptive HIS-CS image fusion. The fusion results of different methods are as shown in Figure 4:

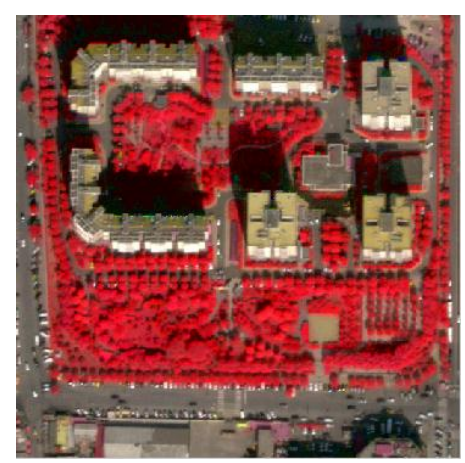

(c) HIS transformation 


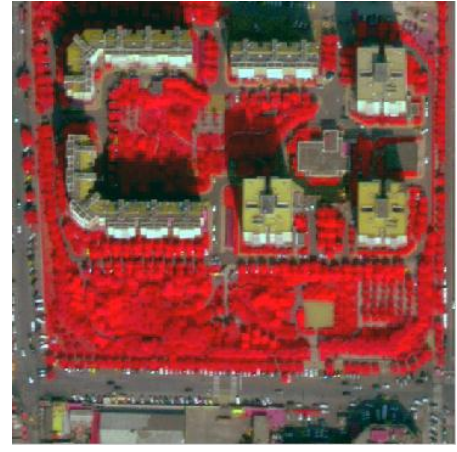

(d) CS image fusion

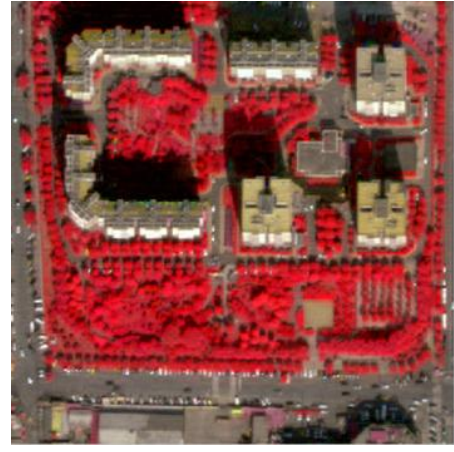

(e) HIS-CS image fusion

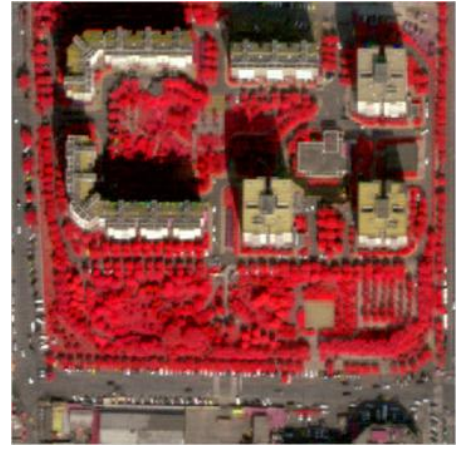

(f) method in this paper

Fig 4 The results of different fusion methods

\section{Evaluation and Analysis of Image Fusion Results}

By comparing the fusion image of different methods of Figure 4, we can find out that there is obvious improvement of the spatial resolution of each method, but there are differences between the improvement degrees of various algorithms. The common feature of the fusion images is that the spectral information has been changed, but the methods of this paper and the
CS image fusion have the smallest spectral changes, the color information is mostly consistent with the original images. So in terms of spectral information retention, the methods of this paper and CS image fusion is better than the traditional methods. But the method of this paper has a better spatial resolution improvement than the method of CS image fusion.

\begin{tabular}{|l|l|l|l|l|l|}
\hline & AG & EN & DD & PSNR & STD \\
\hline PCA & 6.8018 & 11.1301 & 55.9768 & 21.5463 & 50.5000 \\
HIS & 8.8623 & 13.1488 & 53.3584 & 21.9316 & 57.0659 \\
CS & 6.2396 & 12.5802 & 33.6010 & 20.7519 & 57.4108 \\
HIS-CS & 8.8269 & 10.5890 & 53.2103 & 20.7950 & 56.7286 \\
Proposed method & 9.3711 & 13.8277 & 44.3443 & 25.0350 & 57.7704 \\
\hline
\end{tabular}

Table 1 The Comparison of various evaluation indicators with different fusion methods

The image fusion effect can be adjust by different evaluation indicators, such as Average Gradient (AG), Entropy (EN), Degree of Distortion (DD), Peak Signal to Noise Ratio(PSNR), Standard Deviation (SD) et.al.. The result of different image evaluation indicators is shown as in table 1 . As we can see from data of Table 1 , the value of AG, EN, PSNR, STD of the method in this paper are larger than that of other methods, so the method in this paper has the optimal evaluation indicators. The value of DD of this method is larger than CS image fusion, but is smaller than the other traditional methods. So we can see that the spectral information retention of the proposed method is better than the other traditional methods, but not as well as CS image fusion. Overall, the proposed method has a better performance than the traditional methods.

\section{Conclusion}

In this paper, particle swarm optimization algorithm is used to improve the HIS-CS image fusion algorithm. In the proposed method, we combine a series of fusion weight coefficients $\omega$ as particle swarm, and find the optimal value among them to be the optimal fusion weight coefficient $\omega$, which can make the proposed method have certain self-adaptability. In addition, by controlling the step size, we can get a more optimized result, which can balance the improvement of the spatial resolution and spectral information retention. So that by adjusting the step size, we can make the results to meet our requirements. Overall, the proposed method can improve the fusion effect to a certain extent.
But the proposed method has shortcomings ether. The proposed method performs slightly worse in spectral information retention than that of CS image fusion, though it has shown a great advantages in subjective and objective evaluation indices. In addition, in order to make the fusion weight coefficients $\omega$ more optimal, it will lead to a large computation by setting the step size too small. This needs to be further optimized and improved in the lasting research.

\section{References}

Wang X. Research on Remote Sensing Image Fusion Method Based on Compressed Sensing[D]. Beijing: China University of Geosciences, Beijing, 2015.

Yang J. Research on Image Fusion Method Based on Wavelet Transform [D].Beijing: China University of Geosciences, Beijing, 2014.

Jianwei Liu, Xiangwen Hou. An Improved HSI Image Fusion Algorithm[J]. Journal of Northwestern Polytechnical University | J Northwestern Polytech Univ, 2013, 15(05), pp. 368-371.

TU Te-ming, SU shun-chi, CHYUN Hsuen-chyun. A New Look at HIS-like Image Fusion Methods[J]. Information Fusion, 2001, 2(3): 177-186.

Zhang Y. Problems in the Fusion of Commercial High-Resolution Satellites Images as well as LANDSAT 7 Images and Initial Solutions[J]. Proceedings of the Isprs Cig \& Sdh Joint International 
Symposium on Geospatial Theory Processing \& Applications, 2012, 34(4): 9-12.

Guo J. Research on Satellite Remote Sensing Image Fusion Method Based on Compressive Sensing Theory [D]. Beijing: Beijing Jiaotong University, 2013.

Wan T, Canagarajah N, Achim A, et al. Compressive image fusion[C]. international conference on image processing, 2008: 1308-1311.

[8] Atul. Divekar and O. Ersoy, Image fusion by compressive sensing[C] Proceedings of the $17^{\text {th }}$ International Conference on Geoinformatics, pp. 1-6, Fairfax, Va, USA, August 2009.

[9] Senlin Yang, Guobin Wan, Jinghuai Gao. Remote sensing image fusion based on block compressive Sensing [J]. Application Research of Computers, 2015,
32(01): 316-320

[10] Donoho D L. Compressed sensing[J]. IEEE Transactions on Information Theory, 2006, 52(4):1289-1306.

[11] Gesen Zhang. Research on Compressive Sensing and Some Application Techniques[D]. Harbin: Harbin Engineering University, 2012.

[12] Yujie Xu. Improvement and Application of Particle Swarm Optimization[D]. Nanjing: Nanjing Normal University, 2013.

[13] Jianyong Li. Research on Particle Swarm Optimization Algorithm[D]. Zhejiang : Zhejiang University, 2004. 